\title{
ERRATUM
}

\section{Erratum zu: Komposition und Interoperabilität von IT-Systemen}

\section{Johannes Reich ${ }^{1}$}

Online publiziert: 8. September 2021

(c) Springer-Verlag GmbH Deutschland, ein Teil von Springer Nature 2021

\section{Erratum zu:}

\section{Informatik Spektrum 2021}

https://doi.org/10.1007/s00287-021-01376-6

In der Originalpublikation des Beitrags kam es leider zu 4 Fehlern.

\section{(1)}

Die zu Abb. 1 gehörende Formel ist dort nicht zu finden und lautet wie folgt:

$$
\left(\begin{array}{c}
q\left(t^{\prime}\right) \\
\text { out }\left(t^{\prime}\right)
\end{array}\right)=\left(\begin{array}{l}
\int^{\mathrm{int}}(q(t), \text { in }(t)) \\
\int^{\mathrm{ext}}(q(t), \text { in }(t))
\end{array}\right) .
$$

\section{(2)}

In Abb. 3 ist der Text zur Abbildung nicht ganz korrekt. Durch die Platzierungsänderung der Teile $a$ und $b$ während des Produktionsprozesses stimmen die Begriffe „links/ rechts“ nicht mehr und müssen durch die Begriffe „oben/ unten" ausgetauscht werden. Der korrekte Text ist demnach:

Die obere Abbildung zeigt 2 Züge, die sich eine gemeinsame Brücke teilen. Es darf sich höchstens 1 Zug gleichzeitig auf der Brücke aufhalten. Um dies zu gewährleisten, kommunizieren beide Züge mit einem Kontroller. Die untere Abbildung zeigt das Zustandsdiagramm des Protokolls, das Zug (schwarz) und Kontroller (rot) miteinander spre-

chen.

Die Online-Version des Originalartikels ist unter https://doi.org/ 10.1007/s00287-021-01376-6 zu finden.

Johannes Reich

johannes.reich@sap.com

1 SAP SE, Dietmar-Hopp-Allee 16, 69190 Walldorf, Deutschland

\section{(3)}

In Abb. 2a muss es im Kasten von $\mathcal{S}_{\text {ges }}$ nicht ,, out $t_{\text {ges }}(t)=2 *$ $i n_{\text {ges }}(t)+5$ “ heißen, sondern ,out $t_{\text {ges }}(t+1)=2 * i n_{\text {ges }}(t)+5$ “.

\section{(4)}

Die korrekte Adresse des Autors lautet: SAP SE, DietmarHopp-Allee 16, 69190 Walldorf, Deutschland

Der Originalbeitrag wurde korrigiert. 